\title{
Bone marrow-derived mesenchymal stem cells migrate to healthy and damaged salivary glands following stem cell infusion
}

\author{
Silke Schwarz ${ }^{1}$, Ralf Huss ${ }^{2}$, Michaela Schulz-Siegmund ${ }^{3}$, Breda Vogel ${ }^{3}$, Sven Brandau ${ }^{4}$, Stephan Lang ${ }^{4}$ \\ and Nicole Rotter ${ }^{1}$
}

\begin{abstract}
Xerostomia is a severe side effect of radiation therapy in head and neck cancer patients. To date, no satisfactory treatment option has been established. Because mesenchymal stem cells (MSCs) have been identified as a potential treatment modality, we aimed to evaluate stem cell distribution following intravenous and intraglandular injections using a surgical model of salivary gland damage and to analyse the effects of MSC injections on the recruitment of immune cells. The submandibular gland ducts of rats were surgically ligated. Syngeneic adult MSCs were isolated, immortalised by simian virus 40 (SV40) large T antigen and characterized by flow cytometry. MSCs were injected intravenously and intraglandularly. After 1, 3 and 7 days, the organs of interest were analysed for stem cell recruitment. Inflammation was analysed by immunohistochemical staining. We were able to demonstrate that, after intravenous injection, MSCs were recruited to normal and damaged submandibular glands on days 1,3 and 7 . Unexpectedly, stem cells were recruited to ligated and non-ligated glands in a comparable manner. After intraglandular injection of MSCs into ligated glands, the presence of MSCs, leucocytes and macrophages was enhanced, compared to intravenous injection of stem cells. Our data suggest that injected MSCs were retained within the inflamed glands, could become activated and subsequently recruited leucocytes to the sites of tissue damage.
\end{abstract}

International Journal of Oral Science (2014) 6, 154-161; doi:10.1038/ijos.2014.23; published 9 May 2014

Keywords: duct ligation; mesenchymal stem cells; submandibular gland injury; xerostomia

\section{INTRODUCTION}

One major cause of xerostomia in patients suffering from head and neck cancer is radiation therapy. Radiation therapy results in irreversible salivary gland tissue damage and loss of saliva. Without the lubricating and protecting functions of saliva, the oral cavity is highly susceptible to infections, including rampant caries and candidiasis, dysphagia, oral pain and discomfort. These complications result in a significantly reduced quality of life for these patients. Between 300000 and 500000 patients undergo radiation therapy each year worldwide, ${ }^{1}$ and as a consequence, most of these patients will experience severe salivary gland hypofunction. Neither the prophylactic use of amifostine $^{2}$ to prevent radiation-induced xerostomia nor symptomatic treatment strategies, such as pilocarpine, have offered satisfactory relief from symptoms. Intensity-modulated radiation therapy holds great promise in reducing xerostomia; ${ }^{3}$ however, it is neither available nor applicable in the majority of cancer patients. In summary, no effective therapy is yet available for salivary gland hypofunction.

The molecular mechanisms underlying acute radiation damage of the salivary glands are not fully understood. ${ }^{4}$ However, it is clear that xerostomia results from acinar cell loss and the subsequent failure to replace these fluid-producing cells. ${ }^{5}$ Recently, we demonstrated a baseline proliferative activity for all five cell types of the salivary glands, ${ }^{6}$ suggesting that the salivary gland epithelium undergoes a continual level of basal regeneration. Although the exact mechanism of salivary gland regeneration has not been established, differentiation from a putative uncommitted stem cell population has long been favoured as a theory. ${ }^{7}$ This stem cell population is believed to reside in the intercalated ducts because these cells are capable of repopulating both acinar and granular duct compartments. ${ }^{8-9}$ We were recently able to isolate mesenchymal stem cells (MSCs) from human parotid and submandibular glands. ${ }^{10}$ Radiation-induced xerostomia is believed to be caused by the destruction of the stem cell reservoir in the salivary glands. Replacement of these stem cells by stem cell infusion could potentially allow for the resumption of salivary gland regeneration.

We previously demonstrated the regenerative potential of stem cells in an ischaemia-reperfusion model in mice by ligation of the femoral artery. ${ }^{11}$ Importantly, this model did not require the ablation of bone marrow and thus actual transplantation of MSCs. Until now, a number of studies have demonstrated the beneficial effects of different

${ }^{1}$ Department of Otorhinolaryngology, Ulm University Medical Center, Ulm, Germany; ${ }^{2}$ Apceth, Munich, Germany; ${ }^{3}$ Institute of Pharmaceutical Technology, University of Leipzig, Leipzig, Germany and ${ }^{4}$ Department of Otorhinolaryngology, University of Duisburg-Essen, Essen, Germany

Correspondence: Professor N Rotter, Department of Otorhinolaryngology, Ulm University Medical Center, Frauensteige 12, UIm 89075, Germany

E-mail: nicole.rotter@uniklinik-ulm.de

Accepted 27 February 2014 
types of stem cells (bone marrow-derived and salivary gland-derived) on salivary gland regeneration in animal models. ${ }^{12-14}$ Comprehensive review articles have been published that detail the use of the different stem cell types. ${ }^{15,16}$ However, the exact mechanisms, as well as the physiological migration characteristics, remain largely unknown.

In this study, we sought to analyse stem cell distribution in a rodent model of salivary gland damage by comparing intravenous (i.v.) and intraglandular infusions of immortalised bone marrow-derived MSCs. Furthermore, we aimed to analyse the inflammatory responses following surgical duct ligation and stem cell administration to obtain greater knowledge of cellular MSC therapies.

\section{MATERIALS AND METHODS}

\section{Cell isolation, propagation and immortalisation}

Syngeneic adult mesenchymal rat stem cells were isolated from the bone marrow of Wistar rats. The mononuclear cells were cultured in tissue culture flasks at a density of $2 \times 10^{4}$ cells per $\mathrm{cm}^{2}$ in Dulbecco modified Eagle medium (DMEM; PAA, Pasching, Austria), supplemented with $10 \%$ fetal calf serum (FCS; Biochrom, Berlin, Germany) and $1 \%$ penicillin/streptomycin (PAA, Pasching, Austria). After $72 \mathrm{~h}$, nonadherent cells were carefully removed. Adherent cells were washed with phosphate-buffered saline (PBS) and were detached using $0.05 \%$ trypsin-ethylenediaminetetraacetic acid (trypsin-EDTA) and replated in fresh medium. Stem cell lines were established as described before. ${ }^{11,17-19}$ Clonality of the cell lines was achieved by immortalisation of the primary cells with simian virus 40 (SV40) large $\mathrm{T}$ antigen, followed by cellular cloning and subcloning. This method was used because it has been well established in our group. ${ }^{11,17-19}$

\section{Flow cytometry}

After expansion, two strains (clones 5 and 7) were analysed by flow cytometry. A total of $0.5 \times 10^{6}$ cells were pelleted and washed in PBS and resuspended in $100 \mu \mathrm{L}$ of $\mathrm{PBS} / 1 \%$ bovine serum albumin (BSA). The cell suspension was first incubated for 15 min at $4{ }^{\circ} \mathrm{C}$ with $10 \%(\mathrm{~V} /$ $V$ ) sheep serum and then for $30 \mathrm{~min}$ at $4{ }^{\circ} \mathrm{C}$ with antibodies against rat $\mathrm{CD}$ markers or with corresponding isotype controls. Antibodies against rat CD29, CD49a, CD49b, CD49d, CD49e, CD54, CD61, CD90 and CD106 were purchased from BD Biosciences (San Jose, CA, USA), CD49d from Serotec (Raleigh, NC, USA) and CD45 and CD31 from Acris (Herford, Germany).

CD29, or beta-1 integrin, participates as a fibronectin receptor in cell adhesion. It has been regarded as a positive marker of MSCs. ${ }^{20-21}$ CD31 is platelet endothelial cell adhesion molecule 1. The absence of CD31 is a typical feature of MSCs. ${ }^{22}$ CD 45 (protein tyrosine phosphatase receptor type $\mathrm{C}$ or leukocyte common antigen) can be found on the surface of differentiated haematopoietic cells, and it plays an important role in signal transduction to $\mathrm{T}$ - and B-cell receptors; therefore, it has been used as negative selective marker. ${ }^{23-24}$ CD49a, or alpha-1 integrin, has been used to isolate MSCs from bone marrow. ${ }^{25-26} \mathrm{CD} 49 \mathrm{~b}$, or alpha-2 integrin, participates in collagen I binding and might play a role in the survival of MSCs on collagen $\mathrm{I}^{8} \mathrm{CD} 49 \mathrm{~d}$ is the alpha-4 integrin subunit that is present in human MSCs under certain culture conditions. ${ }^{27} \mathrm{CD} 49 \mathrm{e}$, or alpha-5 integrin, participates in fibronectin and fibrinogen binding and is considered a typical MSC marker. ${ }^{28-29} \mathrm{CD} 54$ (intercellular cell adhesion molecule-1, ICAM-1) is known to be responsible for MSC interaction in bone marrow stroma with hyaluronan, a major component of extracellular matrix. ${ }^{30} \mathrm{CD} 61$, or integrin beta-3, is believed to be negative in MSCs. ${ }^{31}$ CD90 (thymocyte differentiation antigen-1,Thy-1) is a positive marker recommended by the International Society for Cellular Therapy position statement. $^{24}$ CD106 (vascular cell adhesion molecule 1, VCAM-1) is expressed on activated endothelial cells, and it facilitates adhesion of haematopoietic cells to endothelial cells and subsequently their migration into tissue. Typically, human bone marrow derived mesenchymal stem cells (BMSCs) express CD106. ${ }^{21,23}$

The cells were washed and resuspended in PBS containing sodium azide and BSA. Shortly before obtaining measurements, 20\% (V/V) (7-amino-actinomycin D (7-AAD, $\left.20 \mu \mathrm{g} \cdot \mathrm{mL}^{-1}\right)$ was added to each sample for $3 \mathrm{~min}$ to stain the dead cells, which were then excluded from the evaluation.

All of the measurements were obtained using a FACSCalibur flow cytometer, and the data were acquired using the CellQuest software (both BD Biosciences, San Jose, CA, USA). The measurements were evaluated with WinMDI software, version 2.8 (free access at http:// facs.scripps.edu/software.html). A minimum of $1 \times 10^{4}$ viable cells of the population was acquired per data set.

The percentage of positive cells was determined as the percentage of cells having a measured fluorescence greater than that of $99.5 \%$ of the cells that were stained with each associated isotype control. A population was considered positive for a surface marker when the percentage of positive cells for that surface marker was $\geqslant 5 \%$.

\section{In vivo studies}

Unilateral ligation of the submandibular gland main excretory duct was performed as described by Turner et al. ${ }^{32}$ Ethical approval was obtained from the Bavarian government (approval no: 209.1/2112531-55/03). Briefly, 24 Wistar rats were anaesthetized by isofluorane inhalation. The experimental animals were positioned on their backs, and the skin of the neck was shaved and disinfected. A longitudinal incision was made in the midline of the neck, and both submandibular glands were exposed. Then, the main excretory duct was exposed under a microscope. At approximately $2 \mathrm{~mm}$ distant from the right gland, the duct was ligated twice with 8-0 silk. On the left side, we performed sham surgery with exposure of the duct only. No ligation was applied on the left side; thus, it served as an internal control.

Subsequently, the skin was sutured with 5-0 Prolene, and the rats were allowed to recover. Approximately $5 \mathrm{~h}$ after the initial damage, 12 animals were i.v. injected in the tail vein with $1 \times 10^{6}$ stem cells per $25 \mathrm{~g}$ of bodyweight. The cell concentration was $10 \times 10^{6}$ cells per $\mathrm{mL}$. Twelve animals received direct injections into the ligated glands under i.v. anaesthesia. For this purpose, the suture was re-opened, and the gland was exposed and visualized directly. The number of stem cells was the same as in the intravenous group: $1 \times 10^{6}$ cells per $25 \mathrm{~g}$ of body weight. The cell concentration was again $10 \times 10^{6}$ cells per mL. A $200 \mathrm{~g}$ rat was injected with $0.8 \mathrm{~mL}$ of cell suspension. The injection was placed in the macroscopic centre of the gland.

The animals were euthanized at days 1, 3 and 7. The salivary glands, liver, small intestine, lymph nodes, spleen and thymus were removed for further analyses. Six additional animals were injected intravenously with stem cells without any further manipulation.

\section{Histology and immunohistochemistry}

Samples were fixed in formaldehyde for $24 \mathrm{~h}$ and embedded in paraffin. Sections of $5 \mu \mathrm{m}$ in thickness were obtained using a microtome.

\section{Haematoxylin-eosin staining}

Haematoxylin-eosin (HE) staining was performed to assess the morphological changes in the salivary gland tissue. 


\section{Immunohistochemistry}

Immunohistochemistry was performed using an anti-SV40 antibody (Calbiochem; EMD Millipore, Bilterica, MA, USA) to visualize injected immortalised stem cells. To visualize inflammatory cells in the context of our experimental model, we stained for myeloperoxidase (MPO) (H300; Santa Cruz Biotechnology Dallas, TX, USA) to visualize neutrophils, for CD45 (H-230; Santa Cruz Biotechnology Dallas, TX, USA) to visualize leukocytes and for CD68 (H-255; Santa Cruz Biotechnology Dallas, TX, USA) to detect macrophages.

The heat-fixated sections were deparaffinized and rehydrated. Subsequently, the sections were digested with $1 \%$ hyaluronidase (H3506-100MG, in PBS; Sigma, Steinheim, Germany ) for $20 \mathrm{~min}$. Endogenous biotin was blocked using the Biotin-Blocking System (Dako, Clostrup, Denmark). Immunohistological staining was performed using the EnVision+System-HRP (DAB) (Dako, Clostrup, Denmark), according to manufacturer's instructions.

The primary antibodies were diluted (1:400), added to the sections and incubated for $1 \mathrm{~h}$ in a humidified box at room temperature. Counterstaining of the cell nuclei was performed with Mayer's haematoxylin for $30 \mathrm{~s}$. We performed semiquantitative evaluation using the following ranges of cell numbers per section in $\times 10$ magnification: 0 , no cell; + , less than 10 cells;,$++ 10-30$ cells; +++ , more than 30 cells.

\section{RESULTS}

Morphological and surface characteristics of rat stem cell lines Two distinct clones (nos. 5 and 7) were analysed before in vivo application. Both clones consisted of adherently growing cells with a fibroblastoid morphology, which, upon appropriate stimulation, gave rise to adipocytes and osteoblasts in culture (data not shown). The cell surface phenotype was found to be comparable to the MSCs isolated from other sources. Immunophenotypically, the cell population was found to be positive for CD29, CD44, CD49e, CD54 and CD90 and negative for CD45, CD61, CD31, CD106 and CD49d (Table 1).

\section{Surgical duct ligation resulted in an inflammatory response} accompanied by necrosis

Following surgical duct ligation, the behaviour of the animals, as well as the uptake of diet and water, was unimpaired. There was distinct swelling of the ligated submandibular region visible on the outside on days 1 and 3 after the surgical procedure, which disappeared by day 7 . There were no signs of local infection in any of the animals. HE staining revealed intercellular oedema starting on day 1 , which was associated with an inflammatory reaction. Impairment of acinar cells was detectable from day 3 onwards. By day 7, there was a widespread necrosis with
Table 1 Stem cell clones 5 and 7 expressed a typical marker profile of MSCs

\begin{tabular}{lccc}
\hline & Clone 5 & & Clone 7 \\
\cline { 2 - 2 } CD marker & Staining intensity & & Staining intensity \\
\hline CD45 & - & - \\
CD31 & - & - \\
CD90 & ++ & ++ \\
CD44 & ++ & ++ \\
CD106 & - & - \\
CD54 & ++ & ++ \\
CD29 & ++ & ++ \\
CD49a & + & + \\
CD49b & - & - \\
CD49d & - & - \\
CD49e & ++ & ++ \\
CD61 & - & - \\
\hline
\end{tabular}

$\mathrm{CD}$, cluster of differentiation; MSC, mesenchymal stem cell; SV40, simian virus 40. Flow cytometric analyses of the cell surface markers of clones 5 and 7 derived from rat bone marrow cells transformed with SV40 large T antigen. - , negative; +, positive; ++ , strongly positive.

Table 2 Stem cells migrated to lymphatic organs and salivary glands after intravenous injections

\begin{tabular}{lccc}
\hline Organs & Day 1 & Day 3 & Day 7 \\
\hline Thymus & + & + & 0 \\
Spleen & + & +++ & +++ \\
Lymph node & + & ++ & + \\
Small intestine & + & +++ & +++ \\
Salivary gland & ++ & ++ & ++ \\
\hline
\end{tabular}

SV40, simian virus 40 .

Semiquantitative overview representing the presence of stem cells $\left(\mathrm{SV}_{4} \mathrm{O}^{+}\right)$in different organs following intravenous injection. 0 , no cell; + , less than 10 cells;,$++ 10-30$ cells; +++ , more than 30 cells.

fibrous tissue formation (Figure 1). The retrieved secondary lymphatic organs all had macroscopically normal sizes and morphology.

\section{Distribution of MSCs after intravenous injections}

Although the stem cells were resuspended thoroughly prior to injection, 2 of 12 of the i.v. injected animals died immediately, most likely due to pulmonary embolisms. The remaining animals, as well as the intraglandularly injected animals, tolerated the procedure well.

Stem cells were detected in each of the retrieved lymphatic organs, namely, the thymus, lymph nodes and Peyer's patches of the small intestine, as well as in the spleen starting on day 1 (Table 2). After 3
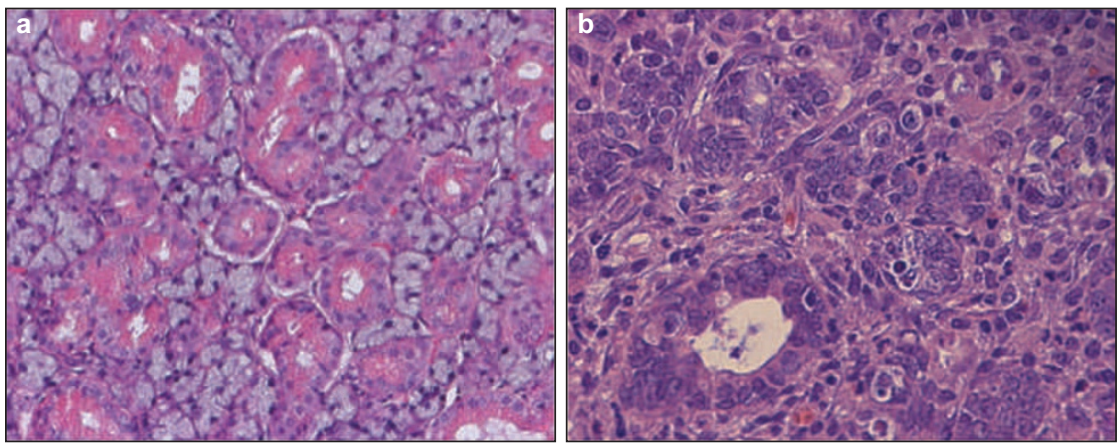

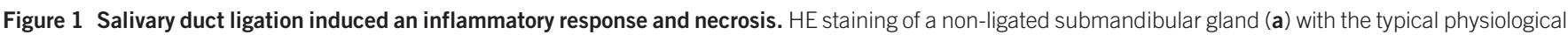
appearance of a normal submandibular gland and (b) a ligated salivary gland showing histological changes following surgical duct ligation for 7 days. Cell nuclei are decentralized, inflammatory cell infiltrate is visible and thickening of the basal lamina can be observed. Magnification $\times 40$. HE, haematoxylin-eosin. 

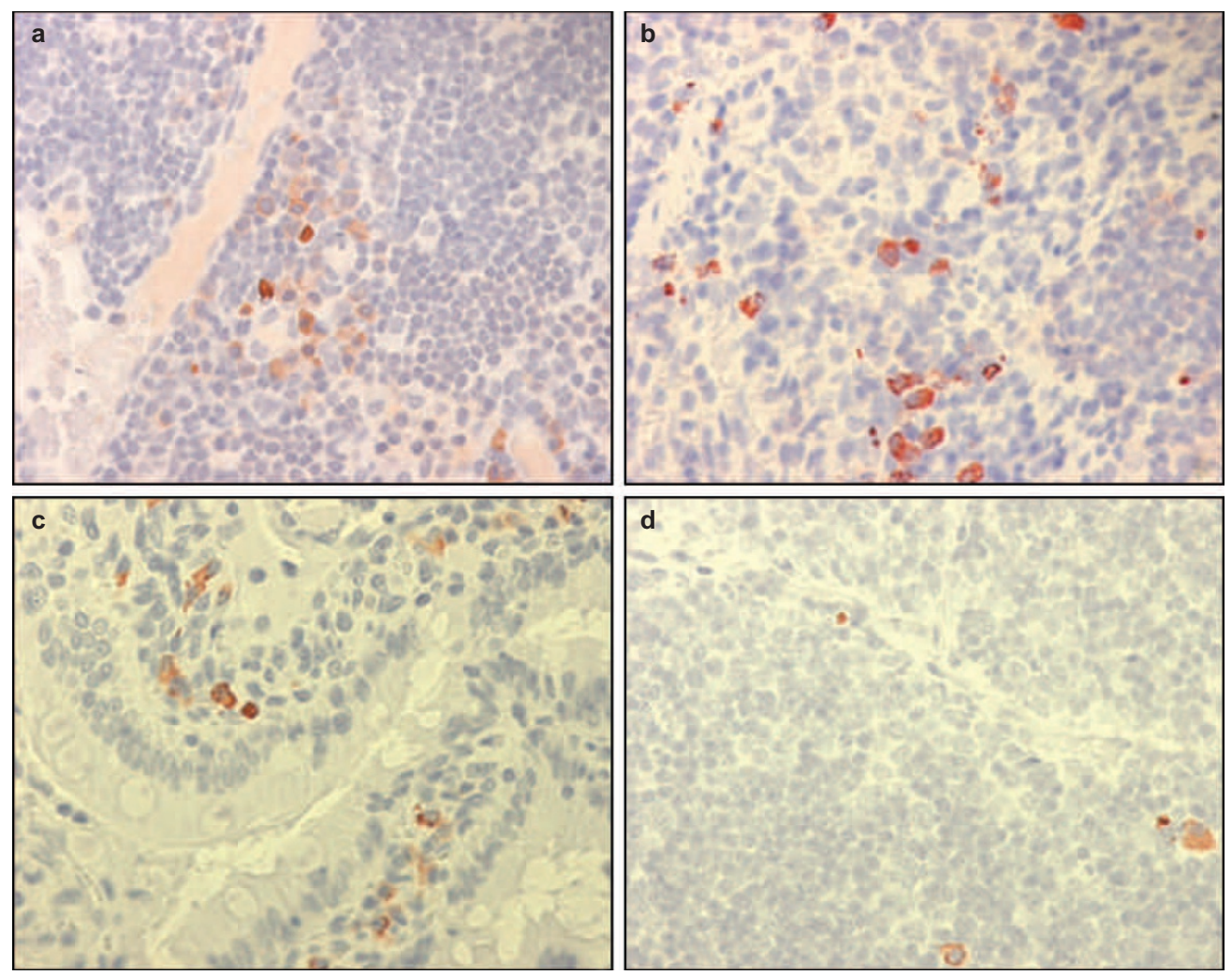

Figure 2 Stem cells were found in lymphatic organs after intravenous injections. Original magnification $\times 40$. Anti-SV40 immunohistochemistry. (a) Lymph node 3 days; (b) spleen 3 days; (c) Peyer's patch of the small intestine 3 days; (d) thymus 3 days. SV40, simian virus 40.

days, a marginal increase in stem cells in the spleen and Peyer's patch was observed (Figure 2). In contrast, there was a decrease in stem cell numbers in some of the lymphoid organs by day 7 (Table 2), such as in the thymus and lymph nodes, while the spleen and Peyer's patch maintained the same levels as observed on day 3 (Figure 2). Starting on day 1 , stem cells were present in the salivary glands (Figure 3). Neutrophils, leucocytes and macrophages were only occasionally present in any of the salivary gland specimens. The numbers of stem cells observed in the respective organs were comparable among the different animals.

\section{Migration of stem cells into ligated and non-ligated salivary glands following intravenous injections}

On day 1 , stem cells were present in ligated salivary glands, infiltrating the periadrenal soft tissue (Figure 4a). In non-ligated glands, MSCs (Figure $4 \mathrm{~b}$ ) were present as well, and the number and distribution of the cells were comparable to those in the ligated glands. Leucocytes and macrophages were only present occasionally in both the ligated and the non-ligated glands. On day 3, diffuse distribution of the SV40-labelled cells was visualized within the ligated (Figure 4c) and non-ligated glands (Figure 4d). Some leucocytes and macrophages were present in a comparable manner. After 7 days, accumulations of MSCs were present in both the ligated and the non-ligated submandibular glands (Figure $4 \mathrm{e}$ and 4f). Again, relatively few macrophages and leucocytes were detectable (Table 3). Surprisingly, neutrophils were almost never detected in any of the specimens at any time point.

\section{Stem cell and leucocyte distributions in ligated and non-ligated salivary glands following intraglandular injections}

Due to the volume of the stem cell solution being equal to the intravenously injected volume $(0.8 \mathrm{~mL})$, significant oedema of the directly
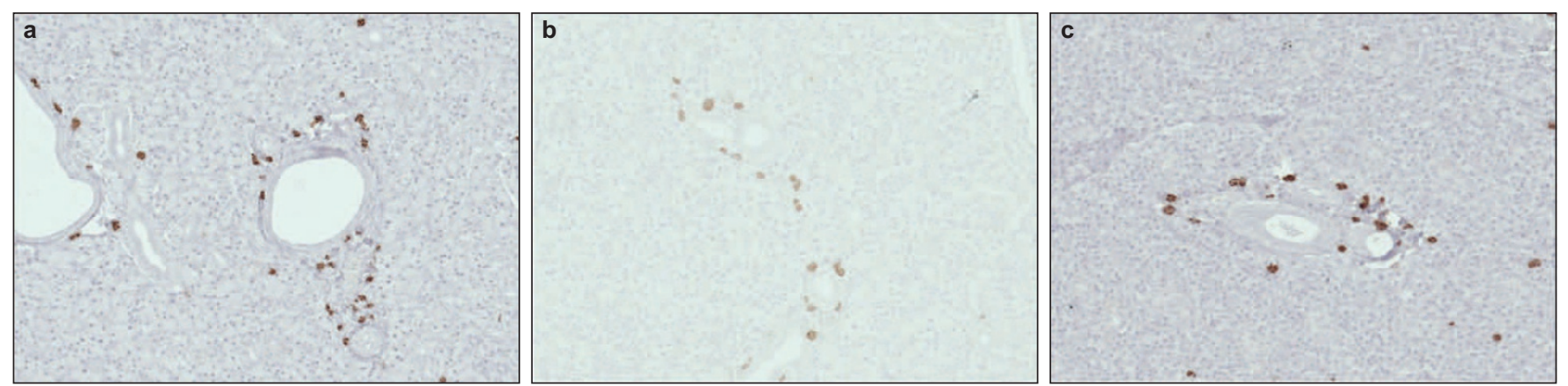

Figure 3 Stem cells migrated to normal salivary glands after intravenous injections. Distribution of stem cells in salivary glands (a) 1 , (b) 3 and (c) 7 days after intravenous injections. In these animals, no further manipulation (surgery) took place. Magnification $\times 10$. 

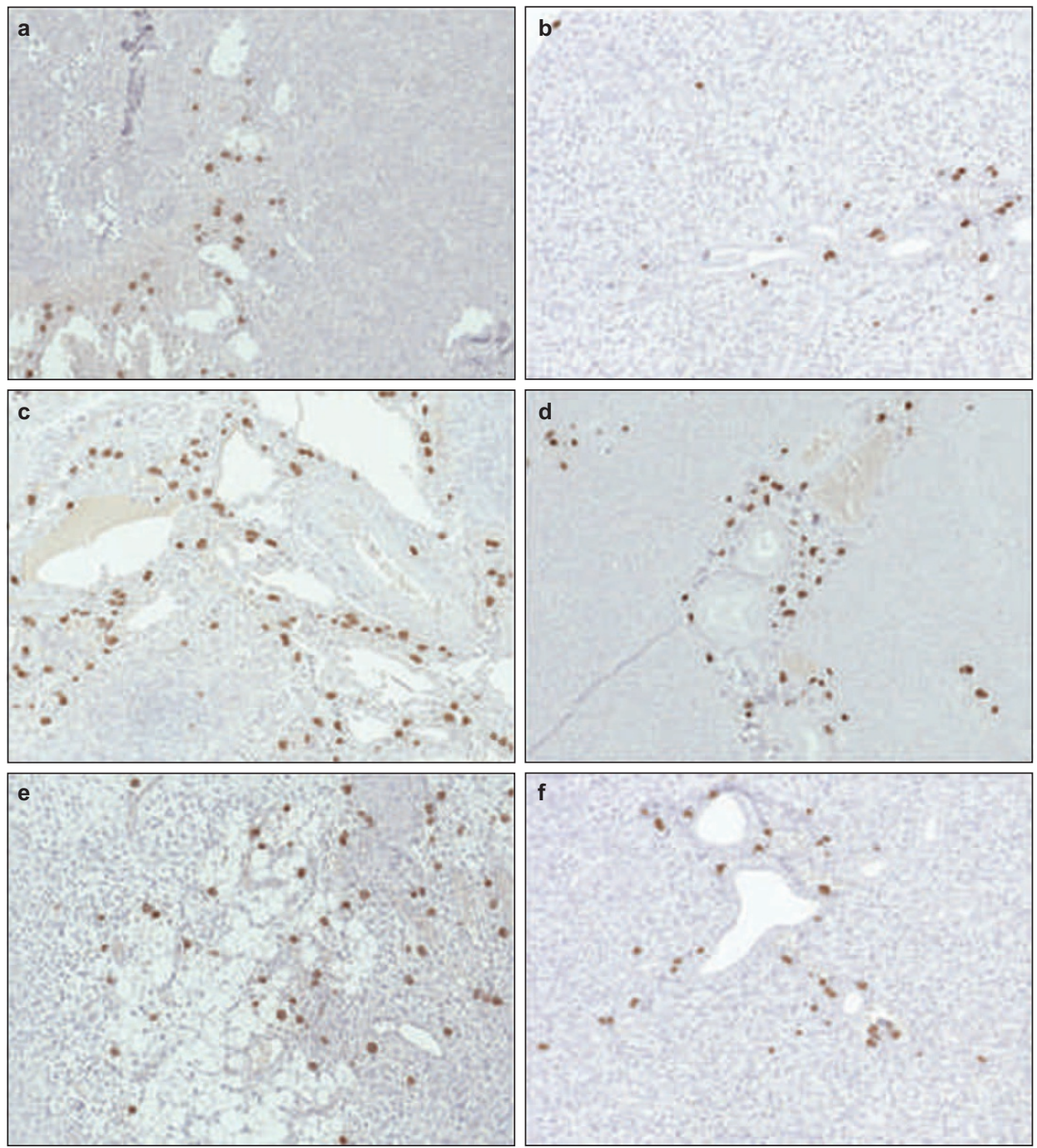

Figure 4 Stem cells migrated to normal and damaged salivary glands after intravenous injections. Distribution of stem cells in ligated (a, c, e) and non-ligated (b, $\mathbf{d}, \mathbf{f})$ glands 1 ( $\mathbf{a}$ and $\mathbf{b}$ ), 3 ( $\mathbf{c}$ and $\mathbf{d}$ ) and 7 (e and $\mathbf{f}$ ) days after intravenous injections. Magnification $\times 10$

injected salivary glands developed. Direct intraglandular injection of stem cells into the ligated salivary gland was performed in comparison to i.v. injection. In contrast to the results obtained by i.v. injection, the cells remained primarily in the region of injection, without significant signs of physiological kinetics. On day 1, a slight influx of MSCs into the soft tissue of the ligated submandibular glands occurred (Figure 5a).
The frequency of MSCs in the ligated glands increased beyond the frequency of MSCs in the sham-operated side. Similarly, recruitment of leukocytes and macrophages to the ligated glands was enhanced (Figure 6). These data suggest that MSCs were preferentially retained in the ligated glands and contributed to the subsequent recruitment of leucocytes to the sites of tissue injury.

Table 3 The presence of leucocytes and macrophages was enhanced after intraglandular injections of MSCs

\begin{tabular}{|c|c|c|c|c|c|c|c|c|c|c|c|c|}
\hline \multirow[b]{2}{*}{ Glands } & \multicolumn{4}{|c|}{ Day 1} & \multicolumn{4}{|c|}{ Day 3} & \multicolumn{4}{|c|}{ Day 7} \\
\hline & Stem cells & CD45 & CD68 & MPO & Stem cells & CD45 & CD68 & MPO & Stem cells & CD45 & CD68 & MPO \\
\hline \multicolumn{13}{|l|}{ Intravenous injections } \\
\hline Ligated glands & ++ & + & + & + & ++ & + & + & 0 & ++ & + & 0 & + \\
\hline Non-ligated glands & + & ++ & + & + & ++ & + & + & 0 & ++ & + & + & 0 \\
\hline \multicolumn{13}{|l|}{ Intraglandular injections } \\
\hline Ligated glands & +++ & + & + & 0 & ++ & +++ & ++ & + & ++ & ++ & + & + \\
\hline Non-ligated glands & + & 0 & 0 & 0 & + & 0 & 0 & 0 & + & 0 & 0 & 0 \\
\hline
\end{tabular}

CD, cluster of differentiation; MPO, myeloperoxidase; MSC, mesenchymal stem cell.

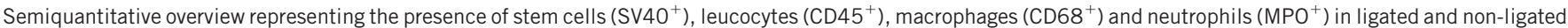
submandibular glands following intravenous and intraglandular stem cell injections. 0 , no cell;,+ less than 10 cells;,$++ 10-30$ cells;,+++ more than 30 cells. 

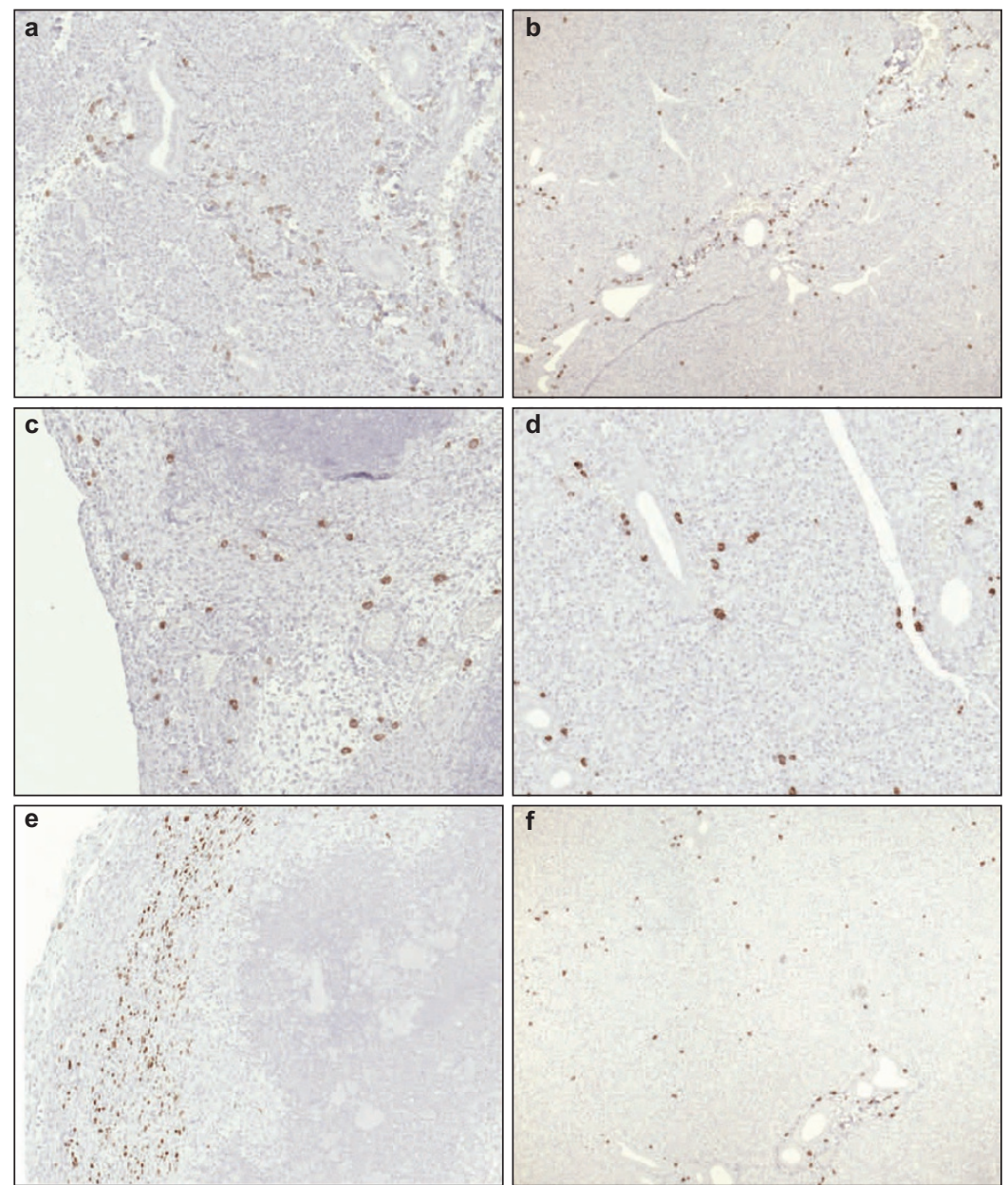

Figure 5 Stem cells were present in normal and ligated salivary glands after intraglandular injections. Distribution of stem cells in ligated (a, c, e) and non-ligated (b, $\mathbf{d}, \mathbf{f}$ ) glands 1 (a and $\mathbf{b}$ ), 3 (c and $\mathbf{d}$ ) and 7 (e and $\mathbf{f}$ ) days after intraglandular injections. Magnification $\times 10$.
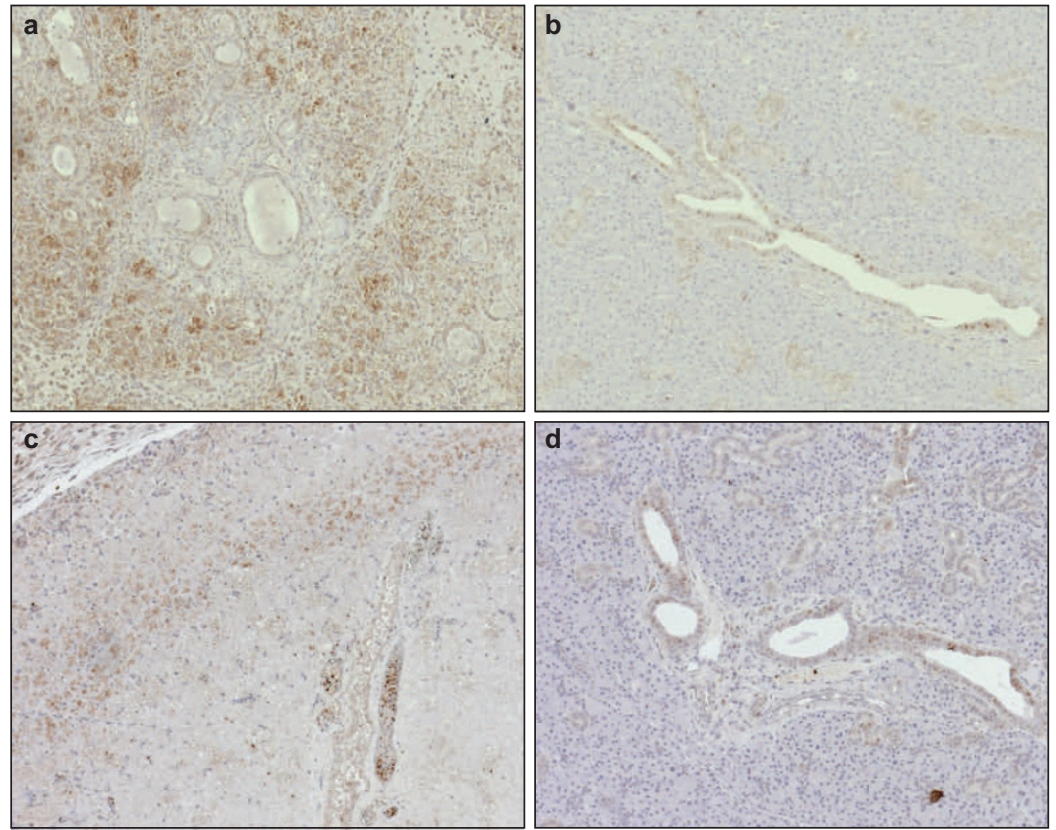

Figure 6 Leucocytes and macrophages preferentially migrated to ligated salivary glands. Enhanced accumulation of leukocytes (a and $\mathbf{b}$ ) and macrophages (c and $\mathbf{d})$ in ligated ( $\mathbf{a}$ and $\mathbf{c}$ ) and non-ligated ( $\mathbf{b}$ and $\mathbf{d})$ salivary glands 3 days after intraglandular stem cell injections. Magnification $\times 10$. 


\section{DISCUSSION}

In this study, we were able to isolate and characterize two MSC lines from rat bone marrow. The cells were immortalized with the SV40 large $\mathrm{T}$ antigen and were cloned, as reported previously. ${ }^{11,33}$ Then, the cells were intravenously injected into a rat model of salivary gland damage, in which the damage was induced by ligation of the main submandibular duct, as described elsewhere. ${ }^{32,34}$ We demonstrated the recruitment of stem cells into the lymphatic organs and trafficking into ligated and non-ligated salivary glands.

MSC recruitment to the spleen, thymus, and lymph nodes in our rat model was comparable to homing, as we previously demonstrated in a mouse model using human cells. ${ }^{33}$ The constitutive expression of the CCR7 ligands CCL21 and CCL19 in lymphatic tissue and the functional expression of CCR7 and CXCR $5^{33}$ have been suggested to play roles in the recruitment of MSCs to lymphatic organs. ${ }^{35-37}$ The injected cells were found as single cells or as clusters throughout the examined organs. The relatively large numbers of injected cells detected in the spleen might have been due in part to trapping by the reticuloendothelial system. ${ }^{33}$

The current observations suggest that there was profound homeostatic/non-inflammatory traffic of MSCs into the salivary glands, which was not significantly enhanced by ligation, at least under the experimental conditions used in this model. This observed traffic was relatively rapid and could already be detected on day 1 , and it did not significantly increase until day 7. Earlier time points, e.g., less than $24 \mathrm{~h}$, were not investigated.

Ligation of the salivary gland can be considered a process of sterile tissue damage. Cell death associated with sterile damage normally induces inflammation and the influx of inflammatory leucocytes. ${ }^{38}$ Surprisingly, however, there was only a slight influx of leucocytes and macrophages in our model, despite ligation of the submandibular duct. This finding indicated that ligation of the duct induced relatively minor trauma only, although induction of interleukin 6 , tumour necrosis factor alpha- $\beta$ and interleukin $1 \beta$ were recently demonstrated at the RNA level. ${ }^{39}$ Leucocytes and macrophages were also detected in untreated salivary glands after i.v. injections of stem cells. However, their presence was clearly enhanced after intraglandular injection of MSCs into ligated glands.

These findings raise the question of why there was such a difference between intravenous MSC and intraglandular MSC injections with regard to subsequent recruitment of CD45- and CD68-positive cells. Based on our findings, we hypothesize that ligation plus the additional trauma caused by direct intraglandular injection could result in enhanced retention and subsequent recruitment of immune cells. This enhanced retention would result in an increased influx of leucocytes. This hypothesis is consistent with our previous investigations, which demonstrated that MSCs activated and interacted with inflammatory cells. ${ }^{40}$ Furthermore, there has been convincing experimental evidence that MSCs recruited monocytes and macrophages. ${ }^{41}$

Neutrophils are mostly involved in antimicrobial responses, and they strongly respond to bacterial stimulation. Accordingly, we recently showed that MSCs from human parotid glands, which were exposed to bacterial lipopolysaccharide, ${ }^{40}$ attracted neutrophils in vitro. In our model of salivary gland damage, only minor sterile inflammation seemed to be induced. Injection of MSCs into this area of mild sterile inflammation resulted in a substantial influx of leucocytes, which was, however, not dominated by neutrophils. This outcome supports recent findings describing a modification of local immune response with absence of neutrophils in vivo. ${ }^{42}$

We previously demonstrated that the injection of a mesenchymal CD34- adult stem cell line showed significant improvement in tissue perfusion in mice with chronic ischaemia. ${ }^{11}$ The mobilisation of stem cells from the bone marrow by granulocyte colony-stimulating factor has been proposed as a similar strategy for repairing infarcted myocardium, as well as salivary gland damage due to radiation therapy. While in the myocardium, the improvement in cardiac function seemed to be a direct effect of granulocyte colony-stimulating factor, rather than induced by the mobilisation of stem cells, ${ }^{43-44}$ in the salivary glands, MSCs mobilized from the bone marrow seemed to contribute directly to the improvements in tissue function and morphology by local cytokine-mediated interactions with the tissue stem cells. ${ }^{45}$ However, the exact mechanism of this improvement has not been clarified completely. Tran and coworkers ${ }^{15}$ postulated that improved salivary gland function resulted from a combination of various mechanisms, such as cell fusion, transdifferentiation, vasculogenesis and paracrine signalling. Importantly, no chemotactic or mobilizing agents were used in the current study, the use of which has often given rise to various side effects and toxicities.

Three other studies have used intraglandular administration of stem cells to date. Kojima and co-workers ${ }^{14}$ used a mouse model, in which they induced salivary gland damage by irradiation with $10 \mathrm{~Gy}$. They used a much smaller number of adipose-derived stem cells $\left(5 \times 10^{5}\right)$ compared to our study, in which we applied $8 \times 10^{6}$ cells in rats. Nevertheless, they were able to demonstrate an increase in salivary flow. Lin and co-workers ${ }^{46}$ used $1 \times 10^{6}$ stem cells in a $15 \mathrm{~Gy}$ irradiated mouse model, and they were also able to demonstrate increased salivary flow rates in irradiated animals. Lim and co-workers $^{47}$ only recently published similar results, confirming the potential of bone marrow-derived stem cells for direct intraglandular injections. Tran and co-workers ${ }^{48}$ aimed to analyse the possible paracrine effects of bone marrow-derived MSCs by injecting so-called 'bone marrow soup', i.e., the intracellular contents of bone marrow-derived stem cells, intraglandularly and intravenously. In fact, they were able to demonstrate that the intracellular contents themselves were as beneficial as the intravenous injection of viable stem cells.

Interestingly, none of these studies investigated whether MSCs migrated to the salivary glands also, in the absence of radiation damage. von Lüttichau ${ }^{33}$ observed the migration of bone marrowderived stem cells into the salivary glands, which was hypothesized to be based on the chemokine receptor profiles of these cells (CCR 4 and CCR10) and their corresponding chemokine expressions in the salivary glands. Therefore, their study, as well as our current study, demonstrated stem cell trafficking into the salivary glands under physiologic conditions (our sham-operated sides and our untreated group). These findings resulted in the conclusion that basic trafficking of MSCs from the bone marrow into the salivary glands occurred under physiological conditions. This conclusion might explain the observations of Tran and co-workers, ${ }^{49}$ who found Y chromosomeexpressing cells in the salivary glands of female patients who had been transplanted with bone marrow from male donors.

Based on the surgical model used, in which the submandibular duct was ligated without removal of the ligation, regeneration was not obtained, and it was not an aim of this study. Therefore, the function of stem cells in the salivary glands could not be assessed. Additionally, the volume of the stem cell suspension used in the study was relatively large. As a consequence, we hypothesize that additional trauma was applied to the salivary tissue structures, thus rendering a direct comparison of both methods of administration more complicated.

\section{CONCLUSIONS}

In conclusion, our data indicated that immortalised stem cells were a useful tool for studying the in vivo fate of MSCs during cellular therapy. Profound homeostatic/non-inflammatory traffic to the salivary 
glands and retention of intraglandularly injected MSCs were observed. These findings could contribute to a greater understanding of the interactions between MSCs and inflammatory cells in future cellular MSC therapies.

\section{ACKNOWLEDGEMENTS}

We thank Dr Dirk Strunk, Medical University of Graz, for providing access to equipment and offering advice regarding flow cytometry. The authors thank Mrs. Sabine Moosmann and Mrs Kathrin Hellberg for their excellent technical assistance. This work was supported by the Novartis Foundation for Therapeutic Research.

1 Vokes EE, Weichselbaum RR, Lippman SM et al. Head and neck cancer. N Engl J Med 1993; 328(3): 184-194.

$2 \mathrm{Ma} \mathrm{C}, \mathrm{Xie} J$, Chen $\mathrm{Q}$ et al. Amifostine for salivary glands in high-dose radioactive iodine treated differentiated thyroid cancer. Cochrane Database Syst Rev 2009; (4): CD007956.

3 Vergeer MR, Doornaert PA, Rietveld DH et al. Intensity-modulated radiotherapy reduces radiation-induced morbidity and improves health-related quality of life: results of a nonrandomized prospective study using a standardized follow-up program. Int J Radiat Oncol Biol Phys 2009; 74(1): 1-8.

4 O'Connell AC. Natural history and prevention of radiation injury. Adv Dent Res 2000; 14: $57-61$

5 Avila JL, Grundmann O, Burd R et al. Radiation-induced salivary gland dysfunction results from p53-dependent apoptosis. Int J Radiat Oncol Biol Phys 2009; 73(2): 523-529.

6 Ihrler S, Zietz C, Sendelhofert A et al. A morphogenetic concept of salivary duct regeneration and metaplasia. Virchows Arch 2002; 440(5): 519-526.

7 Dardick I, Byard RW, Carnegie JA. A review of the proliferative capacity of major salivary glands and the relationship to current concepts of neoplasia in salivary glands. Oral Surg Oral Med Oral Pathol 1990; 69(1): 53-67.

8 Man YG, Ball WD, Marchetti L et al. Contributions of intercalated duct cells to the norma parenchyma of submandibular glands of adult rats. Anat Rec 2001; 263(2): 202-214.

9 Dennis JE, Merriam A, Awadallah A et al. A quadripotential mesenchymal progenitor cel isolated from the marrow of an adult mouse. J Bone Miner Res 1999; 14(5): 700-709.

10 Rotter N, Oder J, Schlenke P et al. Isolation and characterization of adult stem cells from human salivary glands. Stem Cells Dev 2008; 17(3): 509-518.

11 Huss R, Heil M, Moosmann S et al. Improved arteriogenesis with simultaneous skeletal muscle repair in ischemic tissue by $\mathrm{SCL}^{+}$multipotent adult progenitor cell clones from peripheral blood. J Vasc Res 2004; 41(5): 422-431.

12 Lombaert IM, Brunsting JF, Wierenga PK et al. Rescue of salivary gland function after stem cell transplantation in irradiated glands. PLoS One 2008; 3(4): e2063.

13 Sumita Y, Liu Y, Khalili S et al. Bone marrow-derived cells rescue salivary gland function in mice with head and neck irradiation. Int J Biochem Cell Biol 2011; 43(1): 80-87.

14 Kojima T, Kanemaru S, Hirano $S$ et al. Regeneration of radiation damaged salivary glands with adipose-derived stromal cells. Laryngoscope 2011; 121(9): 1864-1869.

15 Tran SD, Sumita Y, Khalili S. Bone marrow-derived cells: a potential approach for the treatment of xerostomia. Int J Biochem Cell Biol 2011; 43(1): 5-9.

16 Pringle S, van Os R, Coppes RP. Concise review: adult salivary gland stem cells and a potential therapy for xerostomia. Stem Cells 2013; 31(4): 613-619.

17 Lange $\mathrm{C}$, Kaltz $\mathrm{C}$. Thalmeier $\mathrm{K}$ et al. Hematopoietic reconstitution of syngeneic mice with a peripheral blood-derived, monoclonal CD34- ${ }^{-}$Sca- $1^{+}$, Thy- $1^{\text {low }}, \mathrm{c}^{-k i t^{+}}$stem cell line. J Hematother Stem Cell Res 1999; 8(4): 335-342.

18 Thalmeier K, Huss R. Highly efficient retroviral gene transfer into immortalized CD $34^{-}$cells and organ distribution after transplantation into NOD/SCID mice. Cytotherapy 2001; 3(4): 245-251.

19 Thalmeier K, Meissner P, Moosmann S et al. Mesenchymal differentiation and organ distribution of established human stromal cell lines in NOD/SCID mice. Acta Haematol 2001; 105(3): 159-165.

20 Sudo K, Kanno M, Miharada K et al. Mesenchymal progenitors able to differentiate into osteogenic, chondrogenic, and/or adipogenic cells in vitro are present in most primary fibroblast-like cell populations. Stem Cells 2007; 25(7): 1610-1617.

21 Chamberlain G, Fox J, Ashton B et al. Concise review: mesenchymal stem cells: their phenotype, differentiation capacity, immunological features, and potential for homing. Stem Cells 2007; 25(11): 2739-2749.

22 Horwitz EM, Le Blanc K, Dominici M et al. Clarification of the nomenclature for MSC: The International Society for Cellular Therapy position statement. Cytotherapy 2005 7(5): 393-395.

23 Pittenger MF, Mackay AM, Beck SC et al. Multilineage potential of adult human mesenchymal stem cells. Science 1999; 284(5411): 143-147.

24 Dominici M, Le Blanc K, Mueller I et al. Minimal criteria for defining multipotent mesenchymal stromal cells. The International Society for Cellular Therapy position statement. Cytotherapy 2006; 8(4): 315-317.

25 Rider DA, Nalathamby T, Nurcombe $V$ et al. Selection using the alpha-1 integrin (CD49a) enhances the multipotentiality of the mesenchymal stem cell population from heterogeneous bone marrow stromal cells. J Mol Histol 2007; 38(5): 449458.

26 Popov C, Radic T, Haasters F et al. Integrins $\alpha 2 \beta 1$ and $\alpha 11 \beta 1$ regulate the survival of mesenchymal stem cells on collagen I. Cell Death Dis 2011; 2: e186.

27 Potapova IA, Brink PR, Cohen IS et al. Culturing of human mesenchymal stem cells as three-dimensional aggregates induces functional expression of CXCR4 that regulates adhesion to endothelial cells. J Biol Chem 2008; 283(19): 13100-13107.

28 Rahman MM, Subramani J, Ghosh M et al. CD13 promotes mesenchymal stem cellmediated regeneration of ischemic muscle. Front Physiol 2014; 4: 402.

29 Benz K, Stippich C, Freudigmann C et al. Maintenance of "stem cell" features of cartilage cell sub-populations during in vitro propagation. J Trans/ Med 2013; 11: 27.

30 Lisignoli G, Cristino S, Piacentini A et al. Hyaluronan-based polymer scaffold modulates the expression of inflammatory and degradative factors in mesenchymal stem cells: involvement of Cd44 and Cd54. J Cell Physiol 2006; 207(2): 364 373.

31 Churchman SM, Kouroupis D, Boxall SA et al. Yield optimisation and molecular characterisation of uncultured $\mathrm{CD} 271^{+}$mesenchymal stem cells in the Reamer Irrigator Aspirator waste bag. Eur Cell Mater 2013; 26: 252-262.

32 Turner JT, Park M, Camden JM et al. Salivary gland nucleotide receptors. Changes in expression and activity related to development and tissue damage. Ann NY Acad Sci 1998; 842: 70-75.

33 von Luttichau I, Notohamiprodjo M, Wechselberger A et al. Human adult CD34 progenitor cells functionally express the chemokine receptors CCR1, CCR4, CCR7, CXCR5, and CCR10 but not CXCR4. Stem Cells Dev 2005; 14(3): 329-336.

34 Takahashi S, Schoch E, Walker NI. Origin of acinar cell regeneration after atrophy of the rat parotid induced by duct obstruction. Int J Exp Pathol 1998; 79: 293301.

35 Campbell DJ, Kim CH, Butcher EC. Chemokines in the systemic organization of immunity. Immunol Rev 2003; 195: 58-71.

36 Forster R, Schubel A, Breitfeld D et al. CCR7 coordinates the primary immune response by establishing functional microenvironments in secondary lymphoid organs. Cell 1999; 99(1): 23-33.

37 Forster R, Mattis AE, Kremmer E et al. A putative chemokine receptor, BLR1, directs B cell migration to defined lymphoid organs and specific anatomic compartments of the spleen. Cell 1996; 87(6): 1037-1047.

38 McDonald B, Kubes P. Cellular and molecular choreography of neutrophil recruitment to sites of sterile inflammation. J Mol Med 2011; 89(11): 1079-1088.

39 Purwanti N, Karabasil MR, Matsuo S et al. Induction of Sca-1 via activation of STAT3 system in the duct cells of the mouse submandibular gland by ligation of the main excretory duct. Am J Physiol Gastrointest Liver Physiol 2011; 301(5): G814G824.

40 Brandau S, Jakob M, Hemeda $\mathrm{H}$ et al. Tissue-resident mesenchymal stem cells attract peripheral blood neutrophils and enhance their inflammatory activity in response to microbial challenge. J Leukoc Biol 2010; 88(5): 1005-1015.

41 Le Blanc K, Mougiakakos D. Multipotent mesenchymal stromal cells and the innate immune system. Nat Rev Immunol 2012; 12(5): 383-396.

42 Georgiev-Hristov T, García-Arranz M, García-Gómez l et al. Sutures enriched with adipose-derived stem cells decrease the local acute inflammation after tracheal anastomosis in a murine model. Eur J Cardiothorac Surg 2012; 42(3): e40e47.

43 Ince $\mathrm{H}$, Nienaber CA. Granulocyte-colony-stimulating factor in acute myocardial infarction: future perspectives after FIRSTLINE-AMI and REVIVAL-2. Nat Clin Pract Cardiovasc Med 2007; 4(Suppl 1): S114-S118.

44 Ince $\mathrm{H}$, Nienaber $\mathrm{CA}$. Future investigations in stem cell activation with granulocytecolony-stimulating factor after myocardial infarction. Nat Clin Pract Cardiovasc Med 2007; 4(Suppl 1): S119-S122.

45 Lombaert IM, Wierenga PK, Kok T et al. Mobilization of bone marrow stem cells by granulocyte colony-stimulating factor ameliorates radiation-induced damage to salivary glands. Clin Cancer Res 2006; 12(6): 1804-1812.

46 Lin $\mathrm{CY}$, Chang FH, Chen CY et al. Cell therapy for salivary gland regeneration. J Dent Res 2011; 90(3): 341-346.

47 Lim JY, Yi T, Choi JS et al. Intraglandular transplantation of bone marrow-derived clonal mesenchymal stem cells for amelioration of post-irradiation salivary gland damage. Oral Oncol 2013; 49(2): 136-143.

48 Tran SD, Liu Y, Xia D et al. Paracrine effects of bone marrow soup restore organ function, regeneration, and repair in salivary glands damaged by irradiation. PLOS One 2013; 8(4): e61632.

49 Tran SD, Redman RS, Barrett AJ et al. Microchimerism in salivary glands after bloodand marrow-derived stem cell transplantation. Biol Blood Marrow Transplant 2011 17(3): 429-433

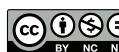

This work is licensed under a Creative Commons AttributionNonCommercial-NoDerivs 3.0 Unported License. The images or other third party material in this article are included in the article's Creative Commons license, unless indicated otherwise in the credit line; if the material is not included under the Creative Commons license, users will need to obtain permission from the license holder to reproduce the material. To view a copy of this license, visit http://creativecommons.org/licenses/ by-nc-nd/3.0/ 